\title{
Imaging Local Dissipation and Magnetic Field in YBCO Films With Artificial Defects
}

\author{
D. V. Abraimov, D. M. Feldmann, A. A. Polyanskii, A. Gurevich, S. Liao, G. Daniels, D. C. Larbalestier,
} A. P. Zhuravel, and A. V. Ustinov

\begin{abstract}
We used Low Temperature Laser Scanning Microscopy (LTLSM) to investigate dc current flow in high- $\mathbf{J}_{c}$ YBCO thin films with artificially prepared defects and correlated the LTLSM response to Magneto Optical Images (MOI). Artificial defects model current blockages such as cracks, high angle grain boundaries or voids. Because the LTLSM voltage response is associated with the local electric field, while MOI shows the local perpendicular magnetic field, the combination of techniques gives complementary local information about the effect of current-limiting defects in superconductors.
\end{abstract}

Index Terms-High-temperature superconductors, laser applications, local electric field, local magnetic field, low temperature scanning laser microscopy, magneto optical imaging.

\section{INTRODUCTION}

$\mathbf{R}$ ECENT progress with coated conductors has been very strong - two US companies have developed continuous processes that are yielding $>10 \mathrm{~m}$ lengths of coated conductors with properties that now approach the best Generation I high temperature superconductor tapes made from $\mathrm{Bi}-2223$ [1], [2]. However, the dominant current-limiting mechanism in these tapes still occurs at grain boundaries, even when the full-width of half-maximum (FWHM) of the texture distribution is only $4-5^{\circ}[3]$.

As was shown recently [4], to understand current limiting mechanisms it is important to correlate the local dissipation distribution with structural defects. In this paper we choose cuts as simple model defects. Using the LTLSM [5]-[8] and MOI [9], [10] techniques correlated to images of surface structure, we clarify the influence of these defects on local dissipation and local magnetic field. Non-uniform flux flow channels occur well above $I_{c}$ even in these quasiideal films and synergistic flux flow near coupled defects is made clear.

\section{EXPERIMENTAL TECHNIQUES}

\section{A. Sample Sets}

To explore the impact of obstructions on current flow, we prepared two test samples. Both are made from similar $250 \mathrm{~nm}$

Manuscript received October 5, 2004. This work was supported by the AFOSR through the MURI Office at UW and by DOE-OETD.

D. V. Abraimov, D. M. Feldmann, A. A. Polyanskii, A. Gurevich, S. Liao, G. Daniels, and D. C. Larbalestier are with University of Wisconsin Applied Superconductivity Center, 927 ERB, Madison, WI 53706 USA (e-mail: abraimov@cae.wisc.edu).

A. P. Zhuravel is with Institute for Low Temperature Physics and Engineering, 310164 Kharkov, Ukraine.

A. V. Ustinov is with Physikalisches Institut III, Universität Erlangen-Nürnberg, D-91058, Erlangen, Germany.

Digital Object Identifier 10.1109/TASC.2005.848679 thick epitaxial YBCO films grown on a single crystal substrate for which MO investigations [11], [12] show that the current flow on scales of a few $\mu \mathrm{m}$ was uniform. The films were deposited on $\mathrm{SrTiO}_{3}$ by pulsed laser deposition at 310 mTorr oxygen pressure and $820^{\circ} \mathrm{C}$, and then annealed in oxygen at 830 Torr and $520^{\circ} \mathrm{C}$ for $30 \mathrm{~min}$. The films were lithographically patterned for four-point measurement and etched by $\mathrm{Ar}$ ions while mounted on a cooled sample mount. Both measured links are $200 \mu \mathrm{m}$ wide and have voltage probes $1 \mathrm{~mm}$ apart. Link \#1 is the same as described in [4]. A photolithographically patterned notch $20 \mu \mathrm{m}$ broad and $1 \mu \mathrm{m}$ wide made a $10 \%$ obstruction of the film width. This cut models current blockages such as cracks, high angle grain boundaries or voids, now recognized to be common in high- $J_{c}$ coated conductors [13]. The transport current presents itself perpendicular to this cut and is forced to deviate around it.

We scribed a more complex obstacle pattern on link \#2 using a focused ion beam (FIB). Defects are vertical cuts of about $1 \mu \mathrm{m}$ width (see Fig. 2(a)) through the YBCO film thickness. The undamaged cross-section at section A is $40 \%$ and $30 \%$ at B.

\section{B. Visualization Techniques}

The LTLSM sample was varnished to a copper cold finger and placed in vacuum in a nitrogen-filled optical cryostat. Temperature was stabilized actively with a precision about $10^{-3} \mathrm{~K}$ so as to provide stability during the $15-40$ minute long scans. Using an adjustable weak thermal coupling between the nitrogen can and the sample holder, we are able to measure for about 1.5 hours without refilling the nitrogen. No magnetic shield or external magnetic field was applied.

The optical part of the LTLSM is placed outside the cryostat. To obtain a Gaussian beam intensity distribution a single mode $4 \mu \mathrm{m}$ fiber was used to connect the diode laser $(\lambda=670 \mathrm{~nm})$ with the scanning laser microscope. Stepping motors move the optical path relative to the cryostat for rough positioning, while a galvanic mirror $\mathrm{x}$-y scanner deflects the probe beam while scanning areas up to $250 \mu \mathrm{m} \times 250 \mu \mathrm{m}$. To achieve high optical resolution at large working distance, we used an infinity-corrected objective with numerical aperture 0.42 . The optical resolution defined as the FWHM of the focused Gaussian laser beam is $1.32 \mu \mathrm{m}$ [14]. We used two lasers; one for scanning and one for pumping. The low power scanning laser was used to initiate the local voltage change response, while the higher-power pump laser was used to create a point-like dynamic defect where desired. The pump diode laser, also with $\lambda=670 \mathrm{~nm}$, was connected by a single mode fiber and deflected by a second set of controllable galvanic $\mathrm{x}-\mathrm{y}$ mirror scanners and focused through 


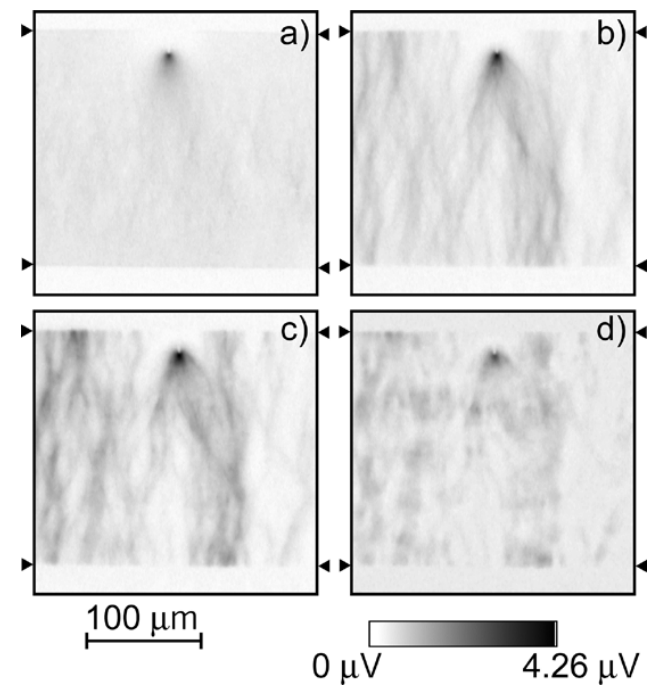

Fig. 1. LTLSM $\delta V$ responses measured on link \#1 with the single cut at top center at constant bias currents corresponding to the same average voltage $\langle V\rangle=3.6 \mathrm{mV}$ at $T=86.6$ (a), 88.7 (b), 89.2 (c), $89.6 \mathrm{~K}$ (d). Macroscopic current flow direction is horizontal.

the same objective lens as the probe laser. The power ratio of about 30 between the two lasers was achieved by adjusting the laser-to-fiber coupling. After reflection from the sample, the light is detected by a video camera and photo detector. It is possible to record a photo image of the scanned area, even while scanning.

The LTLSM voltage change responses $\delta V$ were measured in a standard way [15], [16] at constant bias currents $I_{\mathrm{b}}$ as a function of the laser beam irradiation. To increase the signal-tonoise ratio laser intensity is TTL modulated at the maximum frequency $f=102 \mathrm{kHz}$ of our SR830 Lock-in amplifier and $\delta V$ was measured at the same frequency with time constants up to $30 \mathrm{~ms}$ per point. To measure the voltage response noninvasively we adjusted the intensity of the probing laser such that further decrease of intensity did not change the voltage image pattern. The local heating was so minimal, that critical current $I_{\mathrm{C}}$ did not drop to zero, even while the pump laser was used. LTLSM measurements have been performed at the University of Erlangen-Nuremberg, Germany.

To investigate the flux distribution in this sample, magneto-optical (MO) images were obtained with a Bi-doped YIG film with in-plane magnetization grown on Gadolinium Gallium Garnet (GGG) substrate [9], [10]. The sample was mounted on a cooling finger of a continuous flow optical cryostat located on the X-Y stage of a polarized optical microscope in reflective mode. A digital camera was used to acquire the $\mathrm{H}_{\mathrm{z}}$ component of the field induced in the indicator film placed on the superconductor film surface. Measurements of the magnetic field distribution above the surface of superconductors provide information on the pattern of current flow within the samples.

\section{RESULTS}

The typical $\delta V$ images measured symmetrically around the $10 \%$-obstructed link \#1 at temperatures from 81 to about $87 \mathrm{~K}$ are presented in Fig. 1(a). The dark areas correspond to higher $\delta V$ amplitudes as noted in the scale to Fig. 1. Triangles mark the

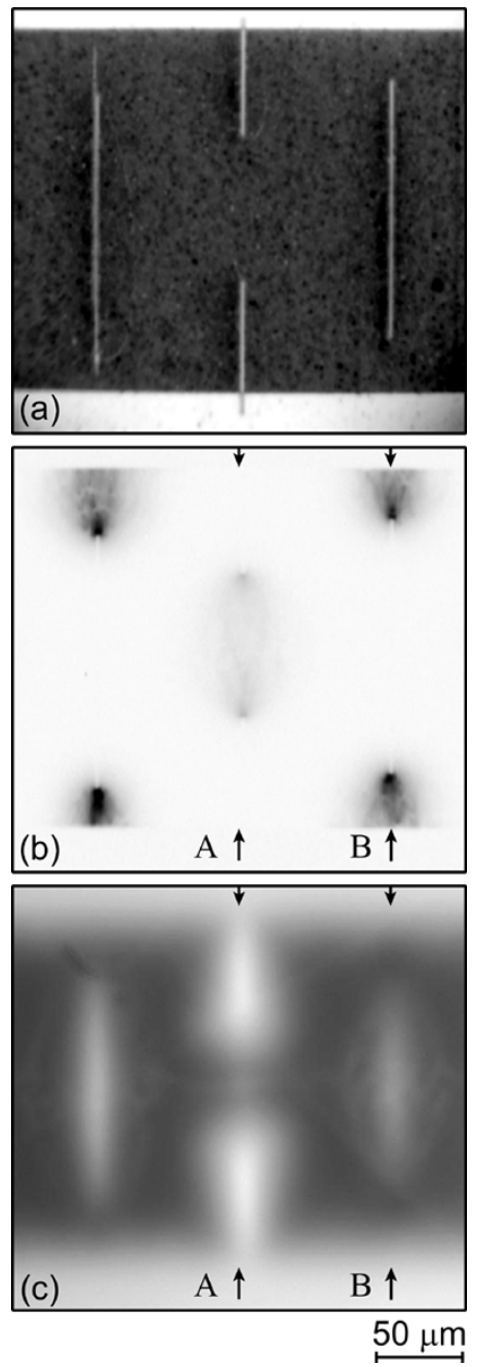

Fig. 2. Images of YBCO thin film with artificial defects. (a) Photo image of sample surface. Light vertical lines in (a) are FIB cuts. (b) $\delta V$ response measured at $T=86.7 \mathrm{~K}, I_{\mathrm{b}}=114 \mathrm{~mA},\langle V\rangle=110 \mathrm{uV}$. The $\delta V$ signal varies from $0 \mu \mathrm{V}$ to $1.95 \mu \mathrm{V}$. Macroscopic current flows horizontally. (c) Zero field cooled MO image measured at $10 \mathrm{~K}$ in $40 \mathrm{mT}$ external magnetic field directed perpendicular to the sample plane. Cross-sections A and B are marked with arrows.

edges of the YBCO film. At temperatures below about $81 \mathrm{~K}$ our links becomes unstable to temperature fluctuations, increasing the risk of burning the link. Therefore we did not measured $\delta V$ below $81 \mathrm{~K}$. We measured $\delta \mathrm{V}$ responses at different $I_{b}$ and did not detect $\delta \mathrm{V}$, when the sample was biased well below $I_{\mathrm{c}}$. The streams of dissipation which emanate from the defect are very striking, showing the great influence that the defect has on the dissipation track. For these images $\langle E\rangle$ is very high, $\sim 36 \mathrm{mV} / \mathrm{cm}$. Nevertheless, even for these single crystal PLD films, there is evidently enough local variation of microstructure to produce locally weaker tracks along which the flux flows preferentially, even far above the conventional $1 \mu \mathrm{V} / \mathrm{cm}$ criterion. At the higher temperatures (Fig. 1(b)-(d)) we do see vortex streams emanating from the high field edges of the strip away from the notch.

The $\delta V$ response of the multi-obstacle link \#2 imaged at lower $\langle E\rangle$ of $1.1 \mathrm{mV} / \mathrm{cm}$ is shown in Fig. 2(b). The dissipation is highly localized at the tips of the FIB cuts in the undamaged 


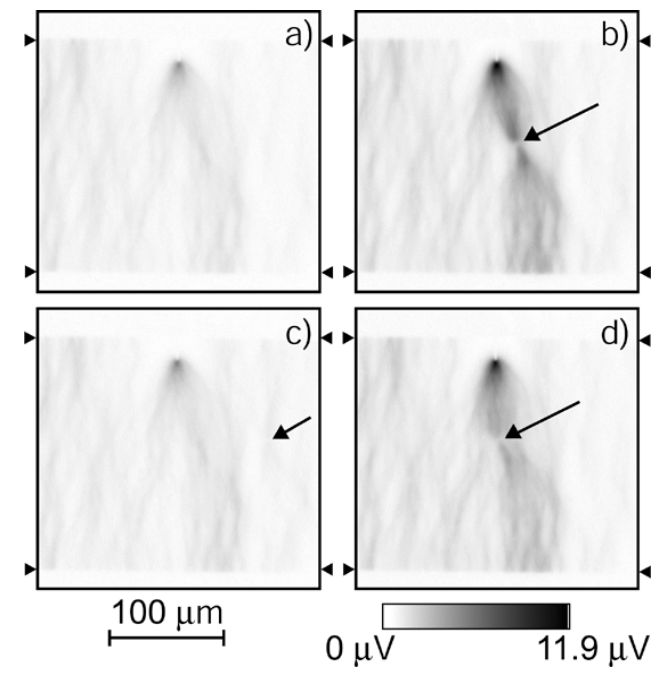

Fig. 3. LTLSM $\delta V$ responses measured on link \#1 with a single cut at bath temperature $88.7 \mathrm{~K}$ and $I_{\mathrm{b}}=142.6 \mathrm{~mA}$. Control image (a) was measured while the pumping beam is off. Images (b), (c), (d) were measured with the pumping beam on. Ratio between scanning and pumping lasers was kept the same during scanning these images. Arrows indicate position of the pumping beam. The $\delta V$ response amplitude scale is the same for all images in this figure. Preferential current flow direction is horizontal.

area. In this case the zone of dissipation is most highly localized in an ellipse emanating from the center cuts through the whole of the residual $15 \%$ sections that lie on either side of the cuts. By contrast the edge-cut obstacles produce much less effect, showing that the average electric field measured at the end of the track is largely produced by the two center cuts. The MO response in Fig. 2(c) provides a complementary view of the influence of the cuts. The image was made by zero-field cooling to $10 \mathrm{~K}$ and then applying a field of $40 \mathrm{mT}$ perpendicular to the film which is above the field of full flux penetration. Light areas correspond to regions of easy flux penetration, whereas dark areas correspond to higher $J_{c}$ regions. Clearly there is no obstacle to penetration along the exterior cuts, but it is also noteworthy that the central cut is partially penetrated too.

As described above, the $\delta V$ response of the single notched link \#1 appears as flux-flow channels lying preferentially in the direction perpendicular to the current flow at temperatures higher then $87 \mathrm{~K}$ (see Fig. 1). Close to $\mathrm{T}_{\mathrm{c}}$ the $\delta V$ response is more complex then described in the model of [4]. $\delta \mathrm{V}$ becomes dependent on both $J_{\mathrm{c}}$ and the local resistivity. We observe that channels do not necessarily take the narrowest that is the notched cross-section, which suggests the importance of the resistivity component for $\delta V$. According to the 1D model of the voltage response [15], the maximum signal appears in areas corresponding to the cross-sections with the smallest $I_{\mathrm{c}}$.

The imaged channels do not change their shape or position during the $\sim 1$ hour of our experiment, nor does change of scanning direction by $90^{\circ}$ or decrease of beam scan rate change the $\delta V$ pattern. However, we have observed broadening of the channels with increasing temperature very close to $T_{\mathrm{c}}$ (Fig. 1).

The pump laser was used to create an additional artificial dynamic point-like defect in link \#1. With 30 times higher intensity, it can locally destroy superconductivity, while still leaving a none zero $I_{\mathrm{C}}$. We see that there is synergistic influence of the permanent $10 \%$ cut and the point-like dynamic defect produced by a varying position but constant-power pump laser, as shown in Fig. 3.

Fig. 3(a) shows an image without the pump laser at rather lower $\langle E\rangle$ than in Fig. 1, such that only small dissipation is seen at the notch. When the pump laser is turned on near the mid strip and just to the right of the notch (Fig. 3(b)), strong dissipation streams appear that are channeled through the pump location. On moving away from the notch (Fig. 3(c)) the vortex channels disappear because the edge notch and the effective center notch do not interact. Moving the pump beam back into the influence zone of the edge notch brings back the strong channel patterns (Fig. 3(d)). No response of the scanned beam is detected in the pump laser focus area, since superconductivity is destroyed there.

\section{DISCUSSION}

Using the same arguments as in [4] we treat our observed $\delta V$ responses as being proportional to the local electric field averaged over the thermal spot $(\sim 3-4 \mu \mathrm{m})$ diameter. The images in Figs. 1 and 2 are indeed very similar to the local electric field distributions calculated in [17], [18]. Given the difficulty of applying the hodograph method to complex geometries, it is valuable to have an experimental tool like the LTLSM that can also image the local $E$ distribution. The case of the multiple-notched sample in Fig. 2(b) is interesting because it is immediately clear that the maximum dissipation is different for cross-sections $\mathrm{A}$ and $\mathrm{B}$. One reason is that the cross-section differs from A to B while a second is that the local self field varies strongly. The undamaged width of $\mathrm{A}$ is $40 \%$, whereas for cross-section $\mathrm{B}$ it is only $30 \%$ of the width. Additionally the self field of the transport current is smaller in the middle of the film and $J_{c}$ is less decreased by self field in the middle of the link. Therefore $I_{\mathrm{c}}$ at cross-section $\mathrm{A}$ is higher than at cross-section B. The highly nonlinear influence of defect size on the dissipation predicted by the calculations of Gurevich and Friesen [17], [18] is very well supported by Fig. 2(b). We can also notice that the MO image can produce a deceiving impression of the impact of the two links. Much greater flux penetration is observed into cross-section A than into B (Fig. 2(c)), but this is an artifact of the fact that the cuts at A are fully open to the external field while those at $B$ are partially shielded by magnetization currents surrounding the slots. Fig. 2 shows that two techniques give complementary information about local fields in the superconducting film.

As seen in Fig. 1(c), the channels of high $\delta V$ response emanate principally from the defect tip, where the cross-section is minimum. Away from the tip, channels diverge as if they repel each other. Dynamic imaging of such effects is shown quite effectively when a second high-intensity pump beam is used to locally destroy superconductivity as in Fig. 3. The LTLSM voltage response has much larger amplitude along the channels that pass through the pumped hot-spot. Using the pump beam, we see that channels connect areas with reduced $J_{\mathrm{c}}$, revealing the lower $I_{\mathrm{c}}$ cross-sections. The strong synergy possible between closely spaced defects is directly shown in this experiment. 


\section{CONCLUSION}

Whereas MO imaging reveals magnetic flux penetration in areas immediately adjacent to defects and on defects itself, the LTLSM $\delta V$ response shows the local dissipation caused by such defects. Such coupled characterizations provide important additional and complementary information about the influence of defects on current transport in 2D superconductors. In these single crystal YBCO films with nominally very uniform microstructure, the LTLSM $\delta V$ responses has shown up stable flux-flow channels which occur at average electric fields well above those used to define $I_{\mathrm{c}}$.

\section{ACKNOWLEDGMENT}

D. V. Abraimov thanks for stimulating discussions G. Logvenov and A. G. Sivakov and thanks O. Turutanov and A. Feofanov for support with the data acquisition program.

\section{REFERENCES}

[1] W. Zhang, X. Li, T. Kodenkandath, D. T. Verebelyi, U. Schoop, C. Thieme, M. W. Rupich, N. Nguyen, E. Siegal, and J. Lynch, "Commercial YBCO coated conductor wire development," in Proc. Mater. Res. Soc. Symp., 2004, EXS-3, EE3.2.1.

[2] V. Selvamanickam, H. G. Lee, Y. Li, X. Xiong, Y. Qiao, J. Reeves, Y. Xie, A. Knoll, and K. Lenseth, "Fabrication of 100 A class, $1 \mathrm{~m}$ long coated conductor tapes by metal organic chemical vapor deposition and pulsed laser deposition," Phys. C, vol. 392-396, pp. 859-862, 2003.

[3] S. I. Kim, D. M. Feldmann, D. T. Verebelyi, C. Thieme, X. Li, A. A. Polyanskii, and D. C. Larbalestier, "Influence of the grain boundary network on the critical current density of deformation-textured MOD $\mathrm{YBa}_{2} \mathrm{Cu}_{3} \mathrm{O}_{7-\mathrm{x}}$ coated conductors," Phys. Rev. B..

[4] D. Abraimov, D. M. Feldmann, A. A. Polyanskii, A. Gurevich, G. Daniels, D. C. Larbalestier, A. P. Zhuravel, and A. V. Ustinov, "Scanning laser imaging of dissipation in $\mathrm{YBa}_{2} \mathrm{Cu}_{3} \mathrm{O}_{7-\delta}$-coated conductors," Appl. Phys. Lett., vol. 85, no. 13, pp. 2568-2570, Sep. 2004.

[5] C. C. Chi, M. M. Loy, and D. C. Cronemeyer, "Optical probing technique for inhomogeneous superconducting films," Appl. Phys. Lett., vol. 40, pp. 437-439, 1982.
[6] V. A. Konovodchenko, A. G. Sivakov, and A. P. Zhuravel, "Visualization of resistive states of superconducting films," Cryogenics, vol. 26, pp. 531-535, 1986.

[7] K. A. Korolev, P. M. Shadrin, J. S. Preston, R. A. Hughes, J. K. Nam, and V. V. Pavlovskii, "Local characterization of HTS thin films by laser scanning microscopy," Phys. C, vol. 341-348, pp. 1435-1438, 2000.

[8] B. E. Klein, S. Seo, C. Kwon, B. H. Park, and Q. X. Jia, "Imaging transport current distribution in high temperature superconductors using room temperature scanning laser microscope," Rev. Sci. Instrum., vol. 73, pp. 3692-3694, 2002.

[9] A. A. Polyanskii, D. M. Feldmann, and D. C. Larbalestier, "Magnetooptical characterization techniques," in Chapter C3.4 of Handbook of Superconducting Materials, D. Cardwell, Ed. Cambridge, U.K.: Univ. Cambridge.

[10] D. Ginley, NREL: IOP, 2003, pp. 1551-1567.

[11] G. Daniels, A. Gurevich, and D. C. Larbalestier, "Impruved strong magnetic field performance of low angle grain boundaries of calsium and oxygen overdoped YBa2Cu3Ox," Appl. Phys. Lett., vol. 77, no. 20, pp. 3251-3253, 2000.

[12] A. Polyanskii, A. Gurevich, A. E. Pashitski, N. F. Heinig, R. D. Redwing, J. E. Nordman, and D. C. Larbalestier, "Magneto-optical study of flux penetration and critical current densities in [001] tilt $\mathrm{YBa}_{2} \mathrm{Cu}_{3} \mathrm{O}_{\mathrm{x}}$ thin-film bicrystals," Phys. Rev. B, vol. 53, pp. 8687-8697, 1996.

[13] D. M. Feldmann, J. L. Reeves, A. A. Polyanskii, G. Kozlowski, R. R. Biggers, R. M. Nekkanti, I. Maartense, M. Tomsic, P. Barnes, C. E. Oberly, T. L. Peterson, S. E. Babcock, and D. C. Larbalestier, "Influence of nickel substrate grain structure on $\mathrm{YBa}_{2} \mathrm{Cu}_{3} \mathrm{O}_{\mathrm{x}}$ supercurrent connectivity in deformation-textured coated conductors," Appl. Phys. Lett., vol. 77, no. 18, pp. 2906-2908, 2000.

[14] A. P. Zhuravel, A. V. Ustinov, D. Abraimov, and S. M. Anlage, "Imaging local sources of intermodulation in superconducting microwave devices," IEEE Trans. Appl. Supercond., vol. 13, no. 2, pp. 340-343, Jun. 2003.

[15] R. Gross and D. Koelle, "Low temperature scanning electron microscopy of superconducting thin films and Josephson junctions," Rep. Prog. Phys, vol. 57, pp. 651-741, 1994.

[16] D. Abraimov, A. G. Sivakov, A. V. Lukashenko, M. V. Fistul, P. Müller, and A. V. Ustinov, "Spatially resolved measurements of critical parameters in superconducting filaments by laser scanning technique," IEEE Trans. Appl. Supercond., vol. 11, pp. 3170-3173, 2000.

[17] A. V. Gurevich and M. Friesen, "Nonlinear transport current flow in superconductors with planar obstacles," Phys. Rev. B, vol. 62, no. 6, pp. 4004-4025, 2000.

[18] M. Friesen and A. Gurevich, "Nonlinear current flow in superconductors with restricted geometries," Phys. Rev. B, vol. 63, pp. 064 521/1-064 521/26, 2001. 